\title{
Design of Semi Physical Simulation Platform for Autonomous Underwater Vehicle
}

\author{
Xu Dong ${ }^{1, a}$, Zhang Lei ${ }^{2, b}$, Ma Shan ${ }^{3, \mathrm{c}}$ and Zhao Jin-xin ${ }^{4, \mathrm{~d}}$ \\ ${ }^{1}$ College of Computer Science and Technology, Harbin Engineering University, Harbin 150001, \\ China \\ $23{ }^{4}$ College of Shipbuilding Engineering, Harbin Engineering University, Harbin 150001, China \\ axudong@hrbeu.edu.cn, dsunny_zhao1984@yahoo.com.cn
}

Keywords: autonomous underwater vehicle; semi physical simulation; hardware architecture; software architecture; intelligent control

\begin{abstract}
To verify the system software logic of a newly designed autonomous underwater vehicle and the hardware architecture, data interface and reliability of the intelligent control system, the semi physical simulation platform was established by combining physical simulation of decision layer with virtual simulation of perception and behavior layer. The hardware and software architecture of the simulation platform were explained in detail. The virtual simulations of motion, sensors and physical simulation of intelligent control system were described. Finally, the obstacle avoidance simulation and long voyage simulation tests were conducted, and the platform is important for the success of sea experiments.
\end{abstract}

\section{Introduction}

As an important instrument and means, autonomous underwater vehicle has been becoming one of the research focuses currently ${ }^{[1]}$, in the field of marine application and ocean developing. Generally, autonomous underwater vehicle is very complicated and expensive, and equipped without umbilical cord. When it works under water, once there is a mistake, a huge damage of time and material will be made, because of the inconvenience in observation and communication. So the stability and reliability must be guaranteed before the actual underwater experiment. But the condition information can not be obtained by sensors through actuators before overall integration. Besides, there is restriction in the instrument working environment, for example, forbidden working in air. They all make physical simulation hardly. The semi physical simulation includes as much instruments as possible, which makes it convenient to test the hardware architecture, software logic, data interface and the reliability of hardware and software architecture in the simulation

On account of the vehicle structure of "OID-I” designed in our laboratory and the special working requirement, the architecture is divided into perception, decision and behavior layer ${ }^{[2-3]}$. In this article, the semi physical simulation platform was established by combining physical simulation of decision layer with virtual simulation of perception and behavior layer.

\section{Hardware Architecture of Semi Physical Simulation Platform}

"OID-I” is cylindrical in shape and about 7.8 meters long. It weights about 6600 kilogrammes. Its regular speed is $5 \mathrm{kn}$, while the maximum speed is $7 \mathrm{kn}$. We divided its architecture into perception, decision and behavior layer. Environment and condition information can be gotten from sensors in the perception layer. Intelligent control is conducted in the decision layer, which also includes algorithm execution, data processing, command transmission. The behavior layer mainly includes the motion system, such as thrusters and control surfaces. The decision layer is the core control system of "OID-I", aimed at planning and controlling the condition and behavior reasonably. There are series of algorithm execution and equipment in this player, and its data interface and reliability need to be verified by simulation urgently. 
Figure 1 shows the hardware architecture of semi physical simulation platform of “OID-I”. Inside the dashed box is virtual simulation, while outside is physical simulation. The virtual simulation includes a graphics work station, a sensor data interface message processor of X86 system, a video converter and a network switch. OCTANE-II from SGI is used as the graphical workstation. OCTANE-II is equipped with a 220v graphics card, 600MHz of CPU R14000TM, 2MB of level 2 cache, 2GB of memory, 36GB of hard disk, two CRT display devices and an IRIX6.5 operation system. It is used for scene simulation, hydrodynamic calculations and the sensor simulation of sound and light vision. The intermediate machine is equipped with a 8 channel PCI serial port card, module ADAM, and it is used to simulate the motion information sensors, such as GPS, Doppler velocimeter, inertial navigator, depth meter, altitude meter and data interface of thrusters. It is of Windows operation system and it communicates with OCTANE-II through TCP/IP protocol.

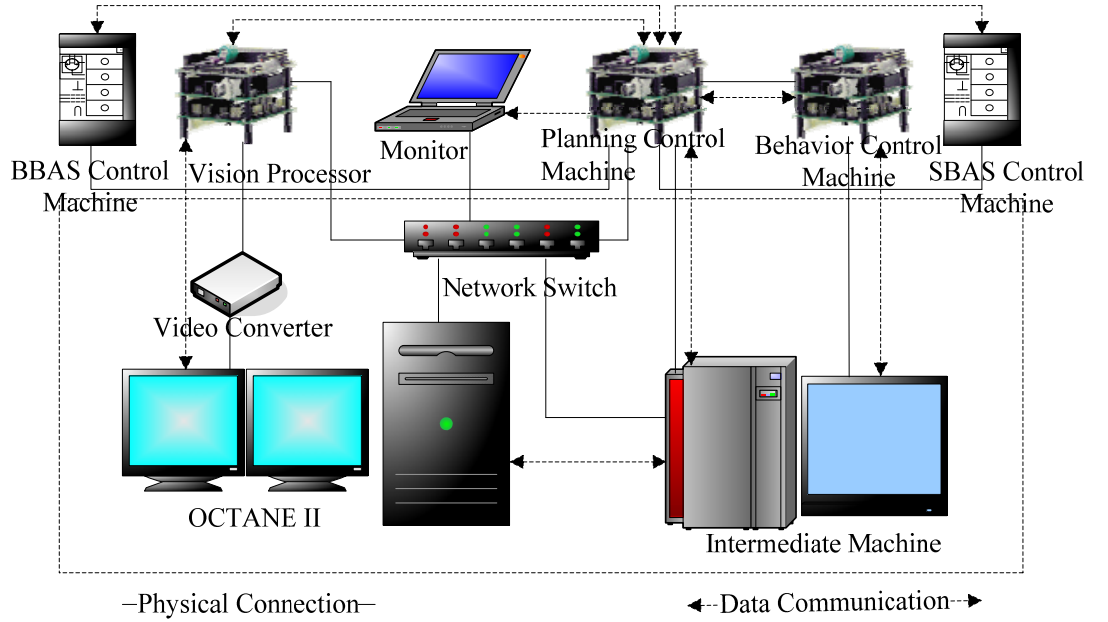

Fig.1 Hardware Architecture of Semi Physical Simulation Platform of "OID-I"

The monitor is used to receive condition information through network and serial port protocol to monitor the working condition and record it for further data analysis. Besides the monitor, outside dashed box, they are all physical simulation sections. The physical simulation is composed of vision processing, planning control, behavior control and buoyancy adjustment system simulation. The vision processing, planning control, behavior control physical simulation platform adopt multi-plate embedded system based on PC104, which comprises a core module integrated with a Pentium III processor, a power source that can provide DC $5 \mathrm{~V}$ and some interface cards for various task requirement. Single chip microcomputer is used in buoyancy adjustment system simulation, including the BBAS and SBAS control machine. The physical simulation system is used for overall path programming, obstacle avoidance, motion control and target identification.

\section{Software Architecture of Semi Physical Simulation Platform}

Motion Simulation of Autonomous Underwater Vehicle

"OID-I" is cylindrical in shape and equipped with two ducted propellers as main thruster and a vertical gear in the tail. In the middle of bow or tail, there is each a channel propeller for vertical propulsion. And a couple of control wings are horizontally put on each side of bow and tail. Considering the vehicle structure and thrust mechanism, with rolling assumed to be negligible in the motion simulation, 5 degrees of freedom simulation model is gotten based on 6 degrees of freedom maneuverability equations ${ }^{[4]}$, by adding the thrusters, control surfaces simulation and environment influence. The hydrodynamic parameters are gotten by combining theoretical calculations, empirical formula estimation and results of model test in the tank. The simulation of thrusters and control surfaces is obtained by results of the actual objects in open water tests. And the consideration of environment influence is based on actual sea, including current and waves near water surface.

When there is current or moving near water surface, the wave first-order and second-order force has important effect ${ }^{[5]}$ on various degrees of freedom of vehicle. We turn the force into force and 
moment of every degree of freedom, so the acceleration of every degree of freedom is calculated. Finally, the motion condition of vehicle is coupled. The overall current distribution map is generated before the simulation. In the simulation, once the vehicle comes into current area, real time reckoning is undertaking. Thus, the motion condition is added with current field function. The International Towing Tank Conference single parameter wave spectrum adopted in the simulation is a standard ocean wave spectrum model, which is recommended by ITTC. The significant wave height is about 1.5 meters, and the corresponding sea condition is level 3-4, which is medium sea condition. The motion equations of vehicle are as follows.

$$
\begin{aligned}
& E A=F_{v i s}+F_{t}+F_{e} \\
& E=\left[\begin{array}{ccccc}
m-X_{\dot{u}} & 0 & 0 & m_{G} & 0 \\
0 & m-Y_{\dot{v}} & 0 & 0 & m_{G}-Y_{\dot{r}} \\
0 & 0 & m-Z_{\dot{v}} & -m_{G}-Z_{\dot{q}} & 0 \\
m_{G} & 0 & -m_{G}-M_{\dot{w}} & I_{y}-M_{\dot{q}} & 0 \\
0 & m_{G}-N_{\dot{v}} & 0 & 0 & I_{z}-N_{\dot{r}}
\end{array}\right]
\end{aligned}
$$

Among them, $E$ denotes mass matrix, including additional mass and additional moment of inertia. $A$ denotes 5 degrees of freedom acceleration matrix. $m$ denotes the mass of "OID-I”. $x_{G}$ and $z_{G}$ are coordinates of gravity center. $I_{y}$ and $I_{z}$ denote rotational inertia to axle Oy and Oz. $X_{\dot{u}} 、 Y_{\dot{v}}$ and $Z_{\dot{q}}$ denote hydrodynamic force derivatives. $F_{v i s}$ denotes viscosity hydrodynamics, and $F_{t}$ denotes the thrust, including thrusters and control surfaces. $F_{e}$ denotes environment force, including current and waves. Runge-Kutta scheme is used to solve the above differential equations, so the motion information in vehicle coordinate system is gotten ${ }^{[6]}$. The conversion matrix between geodetic and vehicle coordinate system is as follows.

$$
\left[\begin{array}{cccccc}
\cos \theta \cos \psi & -\sin \psi & \sin \theta \cos \psi & 0 & 0 & 0 \\
\cos \theta \sin \psi & \cos \psi & \sin \theta \sin \psi & 0 & 0 & 0 \\
-\sin \theta & 0 & \cos \theta & 0 & 0 & 0 \\
0 & 0 & 0 & 0 & 1 & 0 \\
0 & 0 & 0 & 0 & 0 & \sec \theta
\end{array}\right]
$$

$\theta$ denotes attitude angle of pitching, and $\psi$ denotes attitude angle of yawing.

\section{Semi Physical Simulation of Sensors}

Sensors in "OID-I" can be divided into two types according to their function. One is vision sensor related to vision information, and the other is motion sensor related to motion information.

The vision sensors include sound and light vision sensor. The forward looking anti-collision sonar "SeaKing" and side scan sonar "EdgeTech" are put on "OID-I", which make up of a sonar vision system. Because much expertise is needed to simulate sonar in theory, but theory simulation is not much helpful to verify the system reliability, the sonar function simulation is conducted only. A series of rays used for periodic scan denotes sound waves, which simulate the sonar perception of environment and target information. Once any target or obstacle is in the scanned area, the information feedback can be gotten. The light vision system is mainly comprised by an underwater Low Light Level Camera. In the simulation, the workstation scene simulation information is transformed into analog signal by video converter.

Motion sensors mainly include Doppler velocimeter, inertial navigator, GPS, depth meter, altitude meter and etc. The specific simulation of these sensors needs much expertise. To verify the reliability of physical architecture and data interface, data interface message processor is adopted to simulate data interface protocol of motion sensors, in the meantime, the output of sensors is simulated according to actual needs.

Semi Physical Simulation Information Stream of Autonomous Underwater Vehicle 
Software architecture of simulation can be divided into virtual and physical simulation, corresponding to the physical architecture. The virtual simulation comprised motion, scene and vision simulation, while the physical simulation includes simulation of embedded computer, data interface, various information and control stream. Figure 2 shows the semi physical simulation information flow chart of "OID-I". Inside the dashed box is the virtual simulation part, while the physical simulation is outside dashed box.

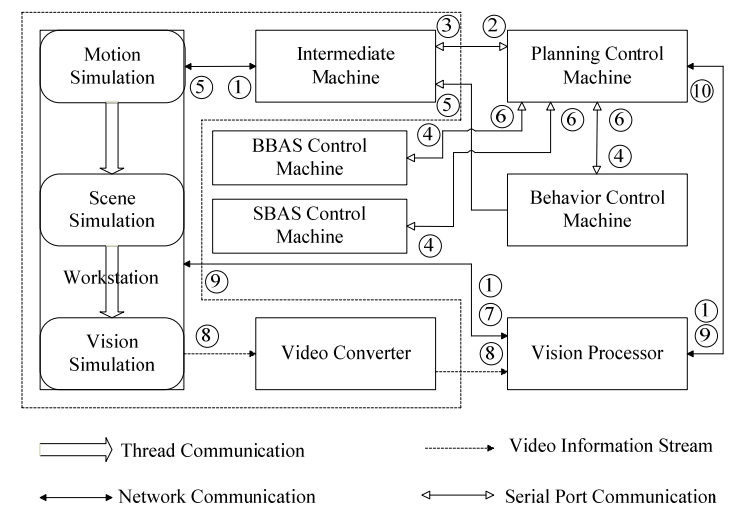

Fig.2 Semi Physical Simulation Information Stream of “OID-I”

OCTANE-II is the physical basis of virtual simulation platform, and it undertakes the majority of simulation calculations. The output of control system is used as the input of simulation software. Dynamic equations are resolved to update the current condition of vehicle. Meanwhile, the corresponding scene information is converted according to interface protocol, to simulate the output of sensors such as anti-collision sonar and Low Light Level Camera in performing the functions. Then the condition and scene information are separately packed to be used as output, which completes the circle of simulation information stream. Intermediate machine simulates data format and interface protocol of various sensors to realize modularity and unification of virtual simulation. It is as shown in the Figure 2.

(1) Results of motion simulation, including the current velocity, pose, depth and height.

(2) Data information of motion sensor processed by data interface message processor, which is mainly the current velocity gotten from Doppler velocimeter, the motion velocity and pose information from inertial navigator and the height from altitude meter.

(3) Data information of DVL processed by data interface message processor, used for transferring to inertial navigator for information correction.

(4) Behavior information related to the next velocity and pose of vehicle, which is decomposed by planning control machine.

(5) Basic command information gotten from behavior control machine.

(6) Feedback information of equipment in behavior layer.

(7) Results of sound vision simulation, mainly including the target scanning data of forward looking sonar "SeaKing" and simulation of side scan sonar "EdgeTech".

(8) Light vision imagines converted by video converter.

(9) Command information that controlling various working condition of sonars.

(10) Information processed by vision processing machine, including position of target and identification results.

\section{Intelligent Control System of Autonomous Underwater Vehicle}

Planning control machine is the core control system of “OID-I", and it is a key module for intelligent behavior. Figure 3 shows the hardware platform of the intelligent control system. It adopts multi-plate embedded system based on PC104, which comprises a core module integrated with a Pentium III processor, a power source that can provides DC 5V, two 8 channel PCI serial port cards and a DIO module based on PC104. The hardware platform uses the embedded real time operation system VxWorks as software environment, which is developed by Wind River. The software system 
includes tasks partitioned according to various function. These tasks are scheduled by VxWorks by priority preemptive scheduling ${ }^{[7]}$.

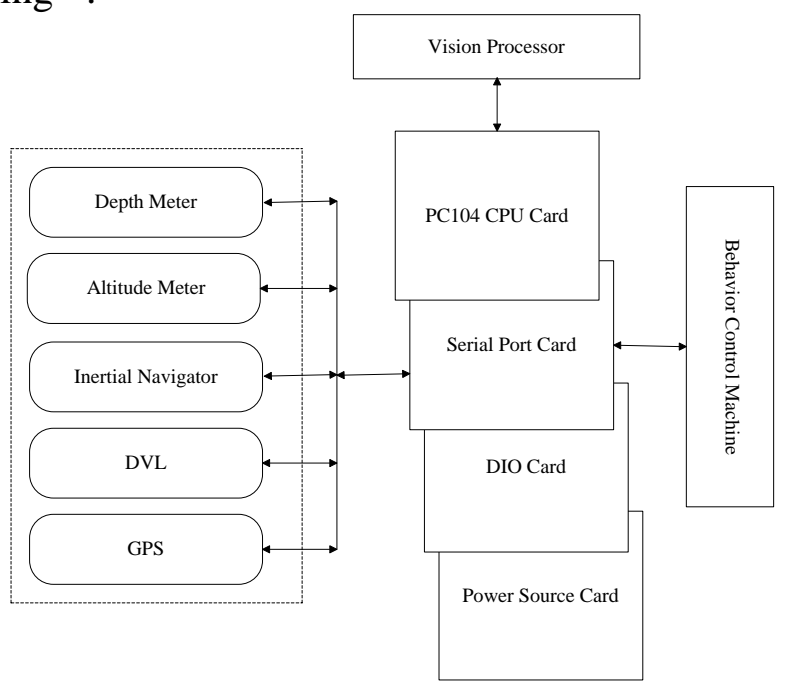

Fig.3 Intelligent Control System of “OID-I”

While the system is running, tasks are divided into 6 various types on the basis of their function.

(1) Acquisition and decomposition of original data

In this section, the original data packet obtained by Doppler velocimeter, inertial navigator, GPS, depth meter, altitude meter and forward looking anti-collision sonar is processed by data interface message processor. And the original data packet is decomposed according to the corresponding data protocol, then we get the original data of system condition.

(2) Condition information decomposition

In this section, the original condition data is preprocessed and conducted smoothing filter, including invalid data elimination and data fusion. The processed data is used as input of intelligent control.

(3) Intelligent control

Estimation of current condition, planning for future condition and output of control instruction are included in this section. The sensor data is preprocessed firstly, then it is analyzed by master control system for condition planning at the next moment. The basic control system gives out planning information according to current condition data and planning system, and decomposes the condition into behavior planning.

(4) Data transfer and command output

Communication in this section is based on network and serial port protocol. Firstly, data gotten from Doppler velocimeter is converted into specific data format that can be received by inertial navigator. Then the data is packed and transmitted to inertial navigator through serial port protocol. Besides, the navigation information is packed and transferred to vision processor through network protocol, as a input for vision processing system. The command output means transferring the result of intelligent controller to behavior control machine through serial port protocol.

(5) Emergency processing

In this section, once there is a communication or hardware fault, emergency behavior will be executed according to the predefined fault type and level. The fault comprises network monitor interruption in experiments with umbilical cord, radio communication interruption above-water, sonar communication interruption underwater and failure of inertial navigator in experiments without umbilical cord. And the emergency behavior includes automatic floating upward, suspension at definite depth and emergency rejection.

(6) System condition record

In this section, the system condition data will be coded, packed and recorded in the form of files for further analysis and post process. 


\section{Results of Simulation Experiments}

Semi physical simulation platform of autonomous underwater vehicle can verify the ability of intelligent control system for long time working conveniently. Not only can the system software logic be tested, but also the hardware architecture, data interface and reliability of the system can be verified. Obstacle avoidance, area search and target identification were conducted in the long voyage simulation experiment. The long voyage involved path programming, motion control and underwater navigation. The experiment route was in reference to the actual map of a sea area in our country. The estimated distance was 158 kilometers, and the maximum speed was $6 \mathrm{kn}$. The simulation duration was about 27.5 hours. Figure 4 shows the recorded results gotten by monitor software in the monitor machine, and the red line denotes the overall track of "OID-I”.

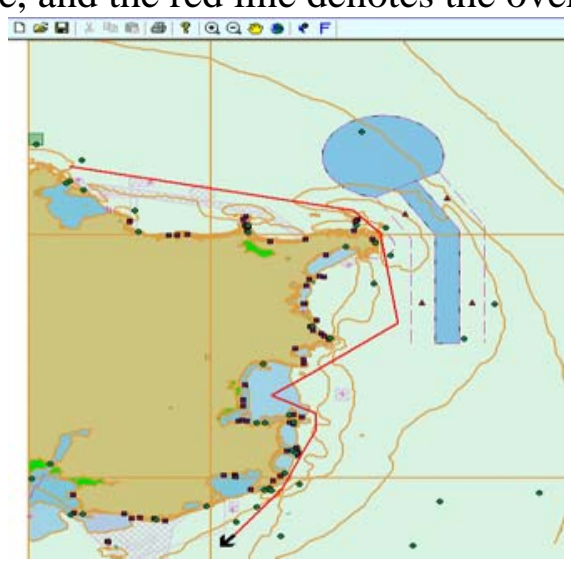

Fig.4 Monitor System of “OID-I”

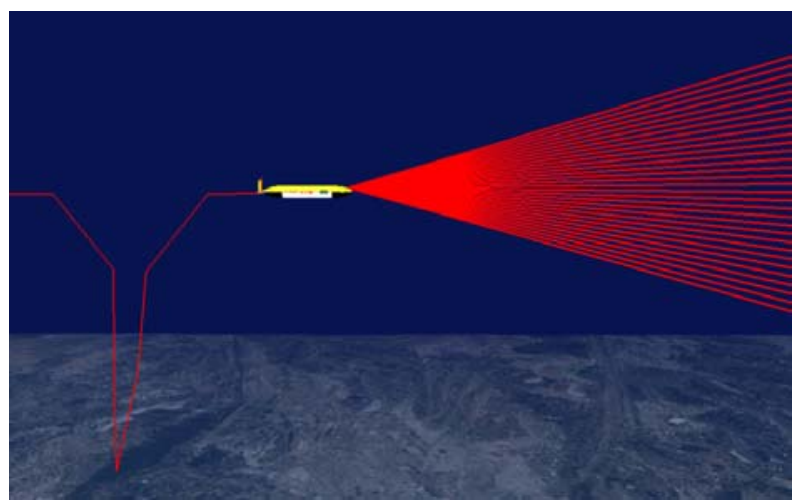

Fig.7 Unpowered Heave Simulation Experiment of “OID-I”

Obstacle avoidance simulation experiment is as shown in Figure 5. In Figure (5a), tracking mode was adopted to observe the experiment process. The visual angle of observer was adjusted in reference to the vehicle in 6 degrees of freedom, and it moved with the vehicle while holding relative attitude. The brown objects were obstacles set by simulation system, and the red line was motion path. The red rays denoted the sound waves from sonar, and it would change into green when any obstacle was detected. In Figure (5b), the vertical view mode was adopted, and the motion path shows the successful obstacle avoidance clearly. The simulation mirrors the ability of obstacle avoidance, which embodies the degree of intelligence of "OID-I".

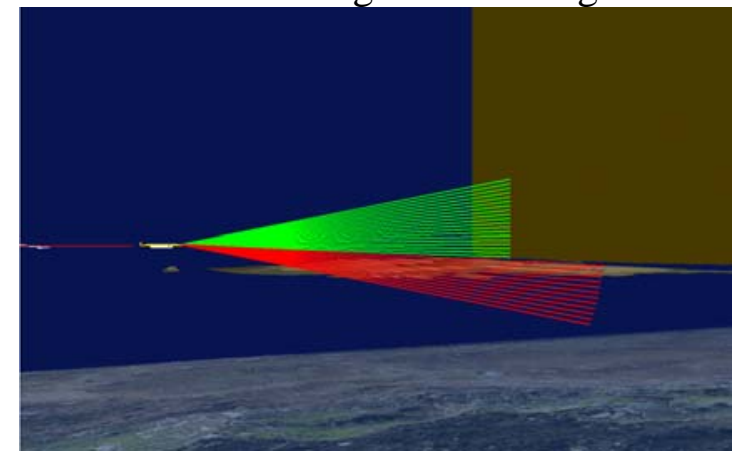

(5a)

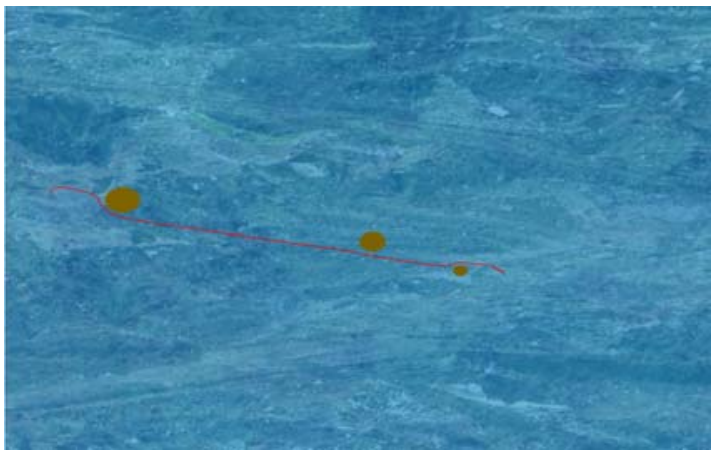

(5b)

Fig.5 Obstacle Avoidance Simulation Experiment of "OID-I”

Figure 6 shows the region search and target identification simulation experiment. Figure (6a) shows the region search path in still water. Figure (6b) shows the region search path with current. Figure (6c) shows target has been searched by "OID-I". Figure (6d) shows the light vision result of target identification. Figure 7 shows the unpowered heave simulation experiment by buoyancy adjustment system. The semi physical simulation experiment lasted a long time and a long distance. Not only did it verify various algorithm, but also tested the data interface and the reliability of hardware architecture. So the simulation provided important reference and guarantee for long voyage in actual sea experiment. 


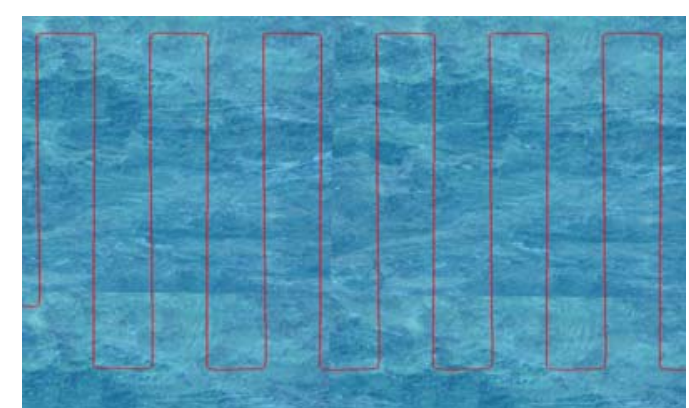

(6a)

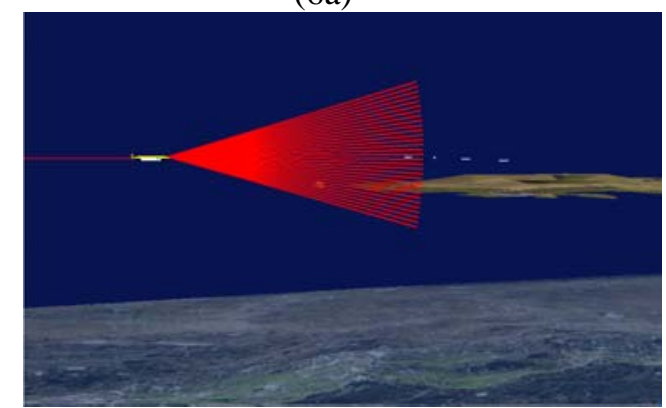

(6c)

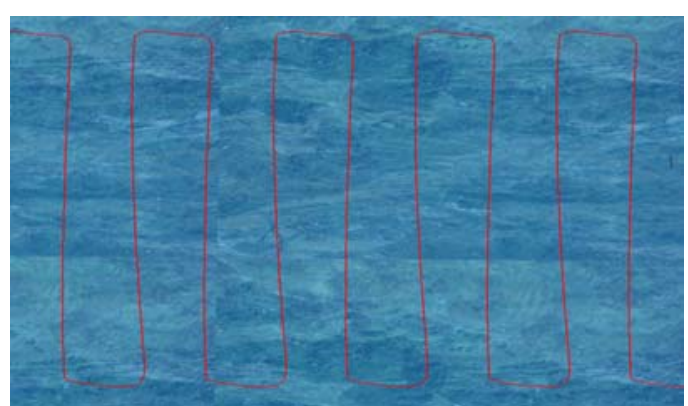

(6b)

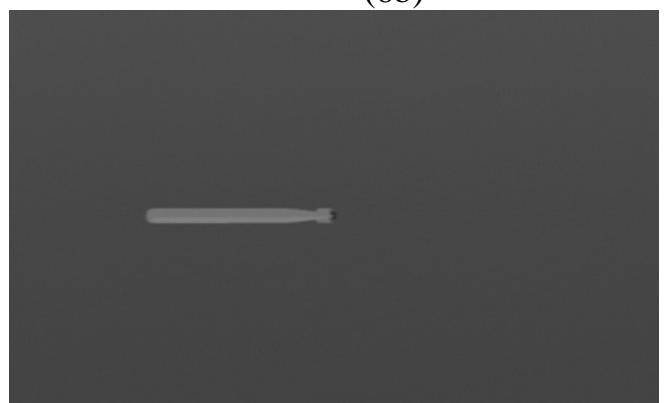

$(6 \mathrm{~d})$

Fig.6 Region Search and Target Identification Simulation Experiment of “OID-I”

\section{Conclusion}

Semi physical simulation platform for autonomous underwater vehicle was established by combining physical simulation of decision layer with virtual simulation of perception and behavior layer. By introducing in the data interface message processor and real intelligent control system, the software logic, hardware architecture of intelligent control system, data interface and reliability of the system were verified in the simulation experiment, which provided credibility for system of autonomous underwater vehicle.

\section{Foundation}

Supported by National Natural Science Foundation of China(51179035) and Fundamental Research Foundation of Harbin Engineering University (Grant No. GK2010260119)

\section{References}

[1] Xu Yuru, Pang Yongjie, Ganyong, SUN Yushan, AUV-state-of-the-art and prospect. CAAI Transactions on Intelligent Systems, 2006, 1(1): 9-16.

[2] Kim T.W., Yuh J. Development of a Real-time Control Architecture for a Semi-autonomous Underwater Vehicle for Intervention Missions. Control Engineering Practice, 2004, 12(12): 1521-30.

[3] Liu Haibo, Gu Guochang, Shen Jing, et al. Neuropsychology-inspired Architecture for AUV. In: Proceedings of 2005 International Conference on Neural Networks and Brain, 2005, 910-914.

[4] Li Ye, Liu Jian-Cheng, Shen Ming-Xue. Dynamics Model of Underwater Robot Motion Control in 6 Degrees of Freedom. Journal of Harbin Institute of Technology, 2005, 12(4): 456-459.

[5] Du Xiao-Xu, Song Bao-Wei, Meng Rui, Li Jia-Wang. Effect of wave to low speed maneuvers of torpedo-like long-distance AUV. Journal of System Simulation. 2008, 20(8):1945-1949.

[6] Du Xiao-Xu, Song Bao-Wei, Pan Guang. Journal of Ship Mechanics, 2011, 15(8):837-843.

[7] Chen Zhiyu, Wen Yanjun, Chen Qi. VxWorks Program Development and Practice. Beijing: Posts and Telecom Press, 2004: 107-229. 